\title{
Digital technique for in vivo assessment of internal and marginal fit of fixed dental prostheses
}

\author{
Park, Ji-Man ; Hämmerle, Christoph H F ; Benic, Goran I
}

\begin{abstract}
Digital approaches to assess the misfit of fixed dental prostheses have been limited to in vitro evaluation. The present article describes a fully digital technique for the in vivo assessment of the fit of fixed dental prostheses by means of a chairside optical scanner and software for 3-dimensional (3D) analysis. The 3D digital capture is performed in 3 steps: an extraoral scan of the restoration, an intraoral scan of the abutment tooth, and an intraoral registration scan of the restoration positioned on the abutment tooth.
\end{abstract}

DOI: https://doi.org/10.1016/j.prosdent.2016.12.016

Posted at the Zurich Open Repository and Archive, University of Zurich

ZORA URL: https://doi.org/10.5167/uzh-136918

Journal Article

Accepted Version

Originally published at:

Park, Ji-Man; Hämmerle, Christoph H F; Benic, Goran I (2017). Digital technique for in vivo assessment of internal and marginal fit of fixed dental prostheses. Journal of Prosthetic Dentistry, 118(4):452-454.

DOI: https://doi.org/10.1016/j.prosdent.2016.12.016 
Digital technique for the in vivo assessment of the internal and marginal fit of fixed dental prostheses

\begin{abstract}
Digital approaches to assess the misfit of fixed dental prostheses have been limited to in vitro evaluation. The present article describes a fully digital technique for the in vivo assessment of the fit of fixed dental prostheses by means of a chairside optical scanner and software for 3dimensional (3D) analysis. The 3D digital capturing is performed in 3 steps: an extraoral scan of the restoration, an intraoral scan of the abutment tooth, and an intraoral registration scan of the restoration positioned on the abutment tooth.
\end{abstract}

\title{
INTRODUCTION
}

The internal and marginal fit of a fixed dental restoration is key in determining its long-term clinical performance. ${ }^{1-3}$ Methods that have been applied to assess the cement space of fixed restorations include direct measurement, cross-sectional measurements, and the impression replica technique. ${ }^{4}$ The replica technique has been the most widely used nondestructive method for the assessment of internal fit in clinical studies. ${ }^{5}$ The accuracy of this method was reported to be similar to that achieved by direct visualization after sectioning of the abutment tooth and prosthesis. ${ }^{6,7}$ Despite being a nondestructive and reliable method for the clinical assessment of restoration fit, the replica technique presents several shortcomings. The number of sections is limited, and their correct placement is challenging with reference to the tooth axis. In addition, the silicone material frequently peels from the internal surface of the restoration during removal 
from the patient's mouth. Moreover, the procedure used for sampling and analysis requires considerable time.

The use of optical scanners can allow more accurate and comprehensive assessment of the restoration than the analog replica technique. Two recent studies reported digital measurement of the restoration fit by using a triple-scan procedure with industrial scanners. ${ }^{8.9}$ The main shortcoming of the method using tabletop scanners is that it can only be applied to in vitro examinations.

The purpose of the present article was to describe a fully digital technique for in vivo assessment of the fit of fixed dental prostheses by means of a chairside scanner and software for 3-dimensional (3D) analysis.

\section{TECHNIQUE}

1. For a single unit, attach a reference structure to the external surface of the restoration (Fig 1). The cubic structure with 2 cylinders at a right angle will act as fiducial marker. Moreover, it will increase the size of the specimen and allow the initiation of the scanning procedure. Trios software (TRIOS 2014-1, v1.3.4.5; 3shape) includes an antiartifact mechanism, which prevents it from scanning objects that occupy less than $50 \%$ of the scan window.

2. Scan the restoration with a chairside scanner (TRIOS 2 Color Cart; 3 shape). The fixed dental prosthesis with the reference structure is attached to a holder stick (Stick'n' Place; Directa) (Fig. 1). Alternatively, a microbrush attached with flowable composite resin can be used. ${ }^{10}$ In an opposing jaw scan mode, the intaglio and external surface of the restoration is scanned. Subsequently, remove the holder stick (Fig. 2A). 
3. In an abutment tooth scan mode, digitize the abutment tooth and adjacent teeth with a chairside scanner (TRIOS 2 Color Cart; 3shape) (Fig. 2B).

4. Make the registration scan of the restoration positioned on the abutment tooth in an occlusion scan mode. This step is done either before or after cementation (Fig. 2C).

5. View the $3 \mathrm{D}$ data sets and verify the relationship between the restoration and the abutment tooth on the computer screen. In case of erroneous matching repeat either the registration scan (step 5) or the entire scanning procedure.

6. Export the 2 scans (restoration and abutment tooth) as stereolithography (STL) files.

7. Import the STL files into a 3D image analysis software (Rapidform v2004; INUS Technology). Evaluate the alignment of the 2 datasets in the cross-sections of the restoration-abutment tooth complex. The intaglio of the restoration should not cross the surface of the abutment tooth (Fig. 3A).

8. Set a cross-section and measure the distance between the intaglio of the restoration and the abutment surface (Fig. 3B).

\section{DISCUSSION}

The digital technique with an intraoral scanner offers a more comprehensive assessment of restoration fit than the analog replica technique. For the digital method, the number of crosssections used for the measurement of distance between the restoration and the abutment tooth is unlimited. In addition, the digital workflow allows the assessment of the thickness of the restoration and the volume of the space between the restoration and the abutment tooth. However, the digital technique for the assessment of the fit of the fixed dental prostheses described in the present article needs validation with regard to accuracy and precision. The present technique can 
be clinically applied to the assessment of fixed restorations with a sufficient number of untreated adjacent teeth that are required for the superimposition of the scans in the triple-scan procedure.

\section{SUMMARY}

The present article describes a fully digital technique for in vivo assessment of the fit of fixed dental prostheses by means of a chairside scanner and software for 3D analysis. The 3D digital capturing is performed in 3 steps: an extraoral scan of the restoration, an intraoral scan of the abutment tooth, and an intraoral registration scan of the restoration positioned on the abutment tooth. This novel assessment technique requires validation. 


\section{REFERENCES}

1. Sorensen SE, Larsen IB, Jorgensen KD. Gingival and alveolar bone reaction to marginal fit of subgingival crown margins. Scand J Dent Res 1986;94: 109-14.

2. Reich S, Kappe K, Teschner H, Schmitt J. Clinical fit of four-unit zirconia posterior fixed dental prostheses. Eur J Oral Sci 2008;116:579-84.

3. Park JM, Hong YS, Park EJ, Heo SJ, Oh N. Clinical evaluations of cast gold alloy, machinable zirconia, and semiprecious alloy crowns: A multicenter study. J Prosthet Dent 2016;115:684-91. 4. Nawafleh NA, Mack F, Evans J, Mackay J, Hatamleh MM. Accuracy and reliability of methods to measure marginal adaptation of crowns and FDPs: a literature review. J Prosthodont 2013;22:419-28.

5. Molin M, Karlsson S. The fit of gold inlays and three ceramic inlay systems. A clinical and in vitro study. Acta Odontol Scand 1993;51:201-6.

6. Rahme HY, Tehini GE, Adib SM, Ardo AS, Rifai KT. In vitro evaluation of the "replica technique" in the measurement of the fit of Procera crowns. J Contemp Dent Pract 2008;9:25-32. 7. Laurent M, Scheer P, Dejou J, Laborde G. Clinical evaluation of the marginal fit of cast crowns-validation of the silicone replica method. J Oral Rehabil 2008;35:116-22.

8. Holst S, Karl M, Wichmann M, Matta RE. A new triple-scan protocol for 3D fit assessment of dental restorations. Quintessence Int 2011;42:651-7.

9. Dahl BE, Rønold HJ, Dahl JE. Internal fit of single crowns produced by CAD-CAM and lostwax metal casting technique assessed by the triple-scan protocol. J Prosthet Dent 2016 Sep 28. [Epub ahead of print].

10. Jogad N, Patil PG, Gade V, Patil S. Alternative technique for handling indirect restorations during evaluation and cementation. J Prosthet Dent 2015;114:458-9. 


\section{FIGURES}

Figure 1. Onlay restoration bonded to reference structure and holder stick. Reference structure in the shape of cube with two cylinders is required for scanning small objects that occupy less than $50 \%$ of scan window. A, Occlusal surface. B, Intaglio surface. C and D, Lateral surfaces.

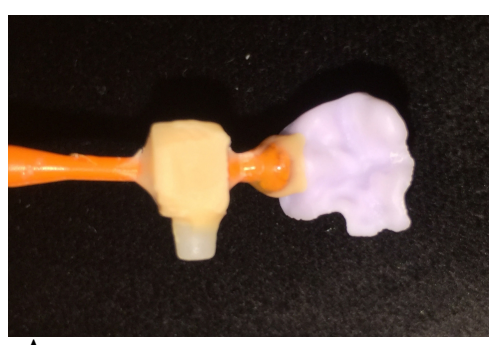

A

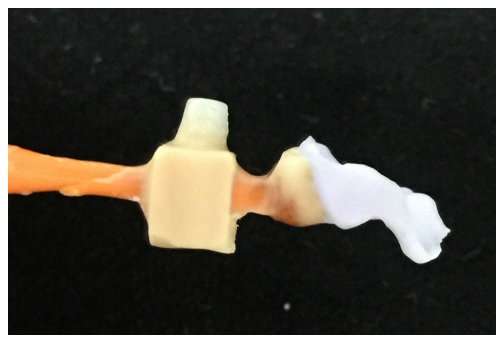

$\mathrm{C}$

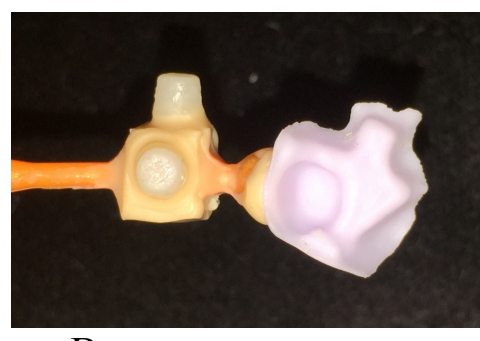

B

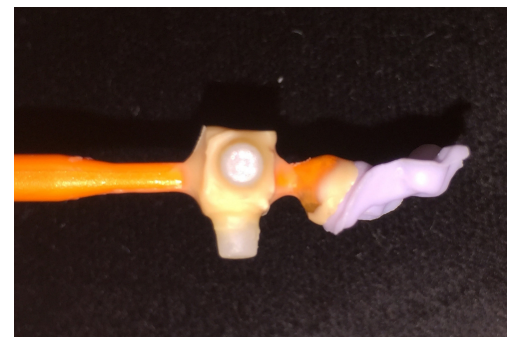

$\mathrm{D}$ 
Figure 2. Triple-scan procedure with chairside scanner. A, Scan of restoration's intaglio and external surface together with reference body. B, Scan of abutment tooth. C, Registration scan of restoration on abutment tooth.
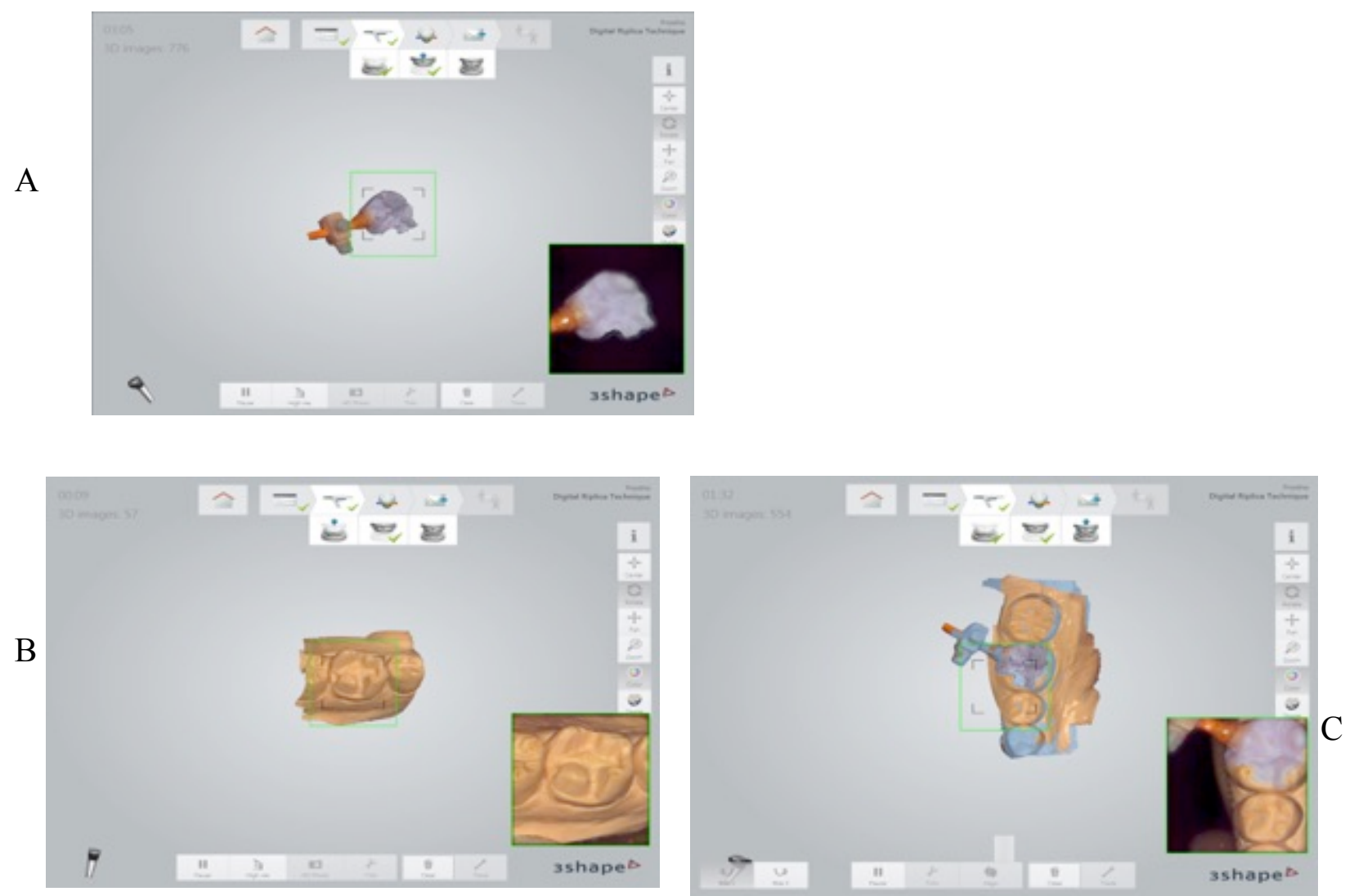
Figure 3. A. Restoration and abutment tooth analyzed with 3D software. B, Mesiodistal crosssection used for distance measurements (arrows) between intaglio surface (orange lines) and abutment tooth. C, Buccolingual cross-section.
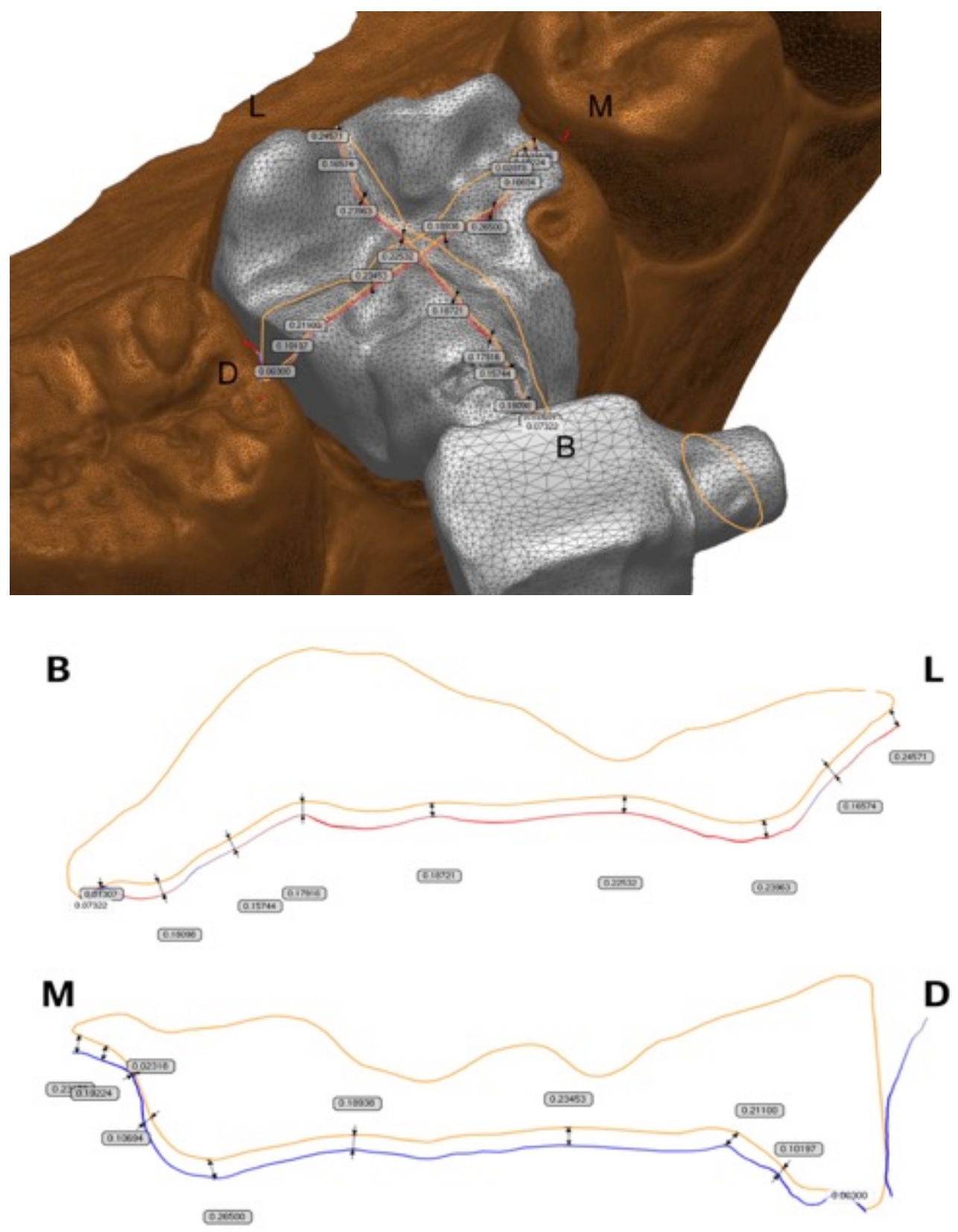